Revue des patrimoines

\title{
L'art selon André Malraux, du Musée imaginaire à l'Inventaire général
}

\author{
Michel Melot
}

\section{(2) OpenEdition}

Journals

Édition électronique

URL : http://journals.openedition.org/insitu/1053

DOI : 10.4000/insitu.1053

ISSN : 1630-7305

Éditeur

Ministère de la culture

Référence électronique

Michel Melot, «L'art selon André Malraux, du Musée imaginaire à l'Inventaire général », In Situ [En ligne], 1 | 2001, mis en ligne le 24 janvier 2012, consulté le 30 avril 2019. URL : http:// journals.openedition.org/insitu/1053; DOI : 10.4000/insitu.1053

Ce document a été généré automatiquement le 30 avril 2019

\section{(c) (i) (9)}

In Situ Revues des patrimoines est mis à disposition selon les termes de la licence Creative Commons Attribution - Pas d'Utilisation Commerciale - Pas de Modification 4.0 International. 


\title{
L'art selon André Malraux, du Musée imaginaire à l'Inventaire général
}

\author{
Michel Melot
}

«L'héritage ne se transmet pas, il se conquiert » André Malraux

1 Lorsqu'il créa l'Inventaire général en 1964, André Malraux pensa-t-il qu'il construisait une formidable machine à mettre à l'épreuve ses propres idées sur l'art?

Figure 1

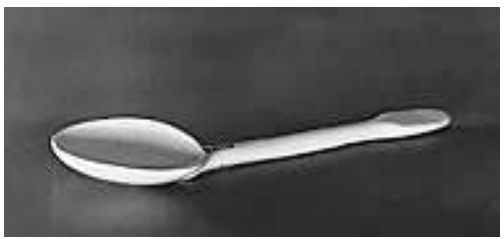


Figure 2

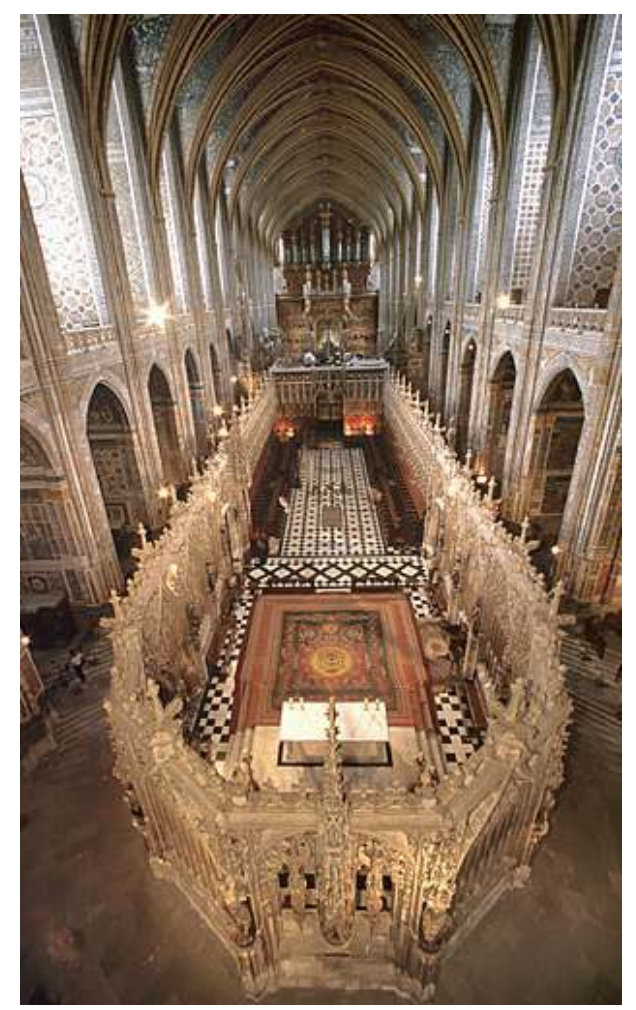

Intérieur de la cathédrale d'Albi

Phot. Inv. Jean-François Peiré @ C Inventaire général, ADAGP, 2000

2 Un abîme, aujourd'hui, semble creusé entre la vision fougueuse, inspirée, d'un art transcendant l'humanité, sublimé dans un petit nombre d'oeuvres exceptionnelles, et la méthode méticuleuse, vétilleuse même, ou notariale d'un relevé sur le terrain d'objets innombrables, le plus souvent communs. Malraux trouve l'art dans la transcendance, l'Inventaire le trouve dans l'immanence. Feuilletez les pages des grandes épopées que sont Les Voix du Silence ou La Métamorphose des dieux, et compulsez ensuite un de ces milliers de "dossiers verts" de l'Inventaire général dans lesquels l'art français est rangé avec un soin entomologique, et vous aurez peine à croire que celui qui a voulu ceci, a écrit cela.

Ecrivant sur l'art, Malraux ne se veut ni historien, ni philosophe. Même s'il intitule une série d'essais "Psychologie de l'art", il n'est pas psychologue. "Ce livre n'a pour objet ni une histoire de l'art (...), ni une esthétique" écrit-il. "Autant prendre La Condition humaine pour un reportage sur la Chine".

4 Le corpus des oeuvres prises en exemples par Malraux, si généreux et varié soit-il, est restreint et se tient dans les limites d'une histoire de l'art la plus académique, où l'art ancien domine, où seuls les noms les plus célèbres ont droit de cité : l'écrivain les manipule d'ailleurs avec jubilation, dans un genre héroïque, comme s'il faisait ruisseler des trésors sous sa plume. Mais l'art modeste, l'art vernaculaire, l'art décoratif ou industriel, celui que rencontre et que fait connaître, la plupart du temps, l'Inventaire général, est absent de son Musée.

5 Non seulement il joue de l'effet de la célébrité des noms à son époque et de son lyrisme littéraire, mais il redouble la rhétorique de son style par une rhétorique de l'image. Il 
exalte les reproductions et leur pouvoir sur la conception même qu'on a de l'art. Malraux fut éditeur avant d'être écrivain. Cadrages, éclairages, qualité de l'impression, mise en page dramatique, discrétion des légendes rejetées en fin d'ouvrage font de ses livres sur l'art des spectacles autant que des textes. Aucun de ces artifices n'a droit de cité à l'Inventaire général, tant dans la rédaction des notices que dans les prises de vue.

Entre l'œuvre d'André Malraux et le propos de l'Inventaire général, y-a-t-il un dénominateur commun, autre que la démesure?

7 Quoi de plus nécessaire, au fond, au "Musée imaginaire"qu'un "Inventaire général" ? Une fois, Malraux s'y laisse prendre et parle de "notre immense inventaire". L'Inventaire général n'est-il pas la "grande réserve" du Musée imaginaire?

L'assimilation du Musée imaginaire à un "inventaire général" vient spontanément sous la plume de Roger Caillois dans sa préface au catalogue de l'exposition "André Malraux" à la Fondation Maeght, en 1973, intitulée : "Esquisse de quelques-unes des conditions requises pour concevoir l'idée d'un véritable Musée imaginaire".

Pour Caillois, Malraux explique l'art d'abord par la confrontation de l'homme avec la mort ("L'homme est le seul animal qui sait qu'il doit mourir"), "d'où l'acharnement de l'écrivain, dit-il, à déceler dans l'art une destination qui dépasse la pure esthétique. "Il ajoute: "Si les Dieux n'ont pas créé l'homme immortel, celui-ci dispose d'un moyen de prouver qu'ils ont été timides, et, par ce biais, de mitiger la malédiction".

Roger Caillois définit ainsi le Musée imaginaire: "Voici l'inventaire général conçu et aménagé dans son économie essentielle : tout le contraire d'une histoire de l'art, plutôt un tableau à entrées multiples des intentions, des continuités, des osmoses, des paroxysmes et des impasses qui en assurent l'unité comme les bifurcations. En même temps, l'échiquier des rapports inextricables des oeuvres avec le climat, la technique, les moeurs, les pouvoirs, l'argent, la foi. Après l'enquête, le patrimoine entier de la planète plonge jusque dans les millénaires de la préhistoire, il annexe tout Kamchatka géographique ou mental, sans compter les résurrections qui métamorphosent. D'où un encombrement, une pléthore qui submerge et décourage (...)". "Notons au passage l'emploi, qui se répand au début des années 1970, du mot "patrimoine" pour englober l'ensemble des biens culturels, au-delà de la sphère traditionnelle des objets d'art, élargissement auquel Malraux, son "Musée" et son "Inventaire" ne furent pas étrangers.

Malraux aussitôt, dans une lettre publiée avec la préface, réfute cette critique d'un Musée imaginaire qui, ouvert à tout, ne servirait à rien: "A votre: "Que faire de lui ?", permettez-moi de répondre d'abord que je voudrais bien savoir ce qu'il fera de nous, et surtout de nos successeurs. "Malraux revendique le caractère illimité de son Musée : "Je crois que chacun y découvrira, qu'il le veuille ou non, son propre Trésor, non un "Kamchatka géographique ou mental". Je crois qu'il pressentira une valeur inconnue, celle qui aura suscité ce trésor (...) C'est pourquoi je ne puis voir dans ce Musée une aventure grandiose et insensée ; pourquoi je ne puis dire : rien ne surnage (...) Ce Trésor existe (...) et peut-être révèlera-t-il à nos successeurs les valeurs qui rassemblent ses oeuvres...". 

Théologique, car elle fait penser à la querelle janséniste: les objets baptisés par une époque pour être élus dans "le monde de l'art" sont-ils prédestinés? Les masques nègres devaient-ils rejoindre les ateliers cubistes ? Le rachat est-il possible? L'accélération du phénomène moderne de "l'art", son investissement dans des objets imprévus, apportentils aujourd'hui, après le ready-made, l'art conceptuel, l'objet industriel et la notion ambiguë de "patrimoine", la réponse que Malraux laisse entendre. A poser la question avec tant de force, il prenait le risque d'une réponse aussi forte : non, l'œuvre d'art, pas plus que le signe, n'existe en soi ; tout objet parlant un autre langage que l'utilitaire, peut entrer dans le Musée imaginaire. redécouvertes, doit admettre l'idée de "réincarnation" des oeuvres, expliquer les réinterprétations, les acculturations d'une civilisation dans une autre, y voit d'abord un effet de ce que Malraux appelle "la corrélation des formes", à laquelle il ne croit pas : "Il devient clair que le Musée imaginaire ne se réduira pas à la parenté de ses formes" et, plus loin : "Notre Musée imaginaire est lié à l'art moderne qui l'accompagne ou le suscite, par des liens plus complexes que ceux de la ressemblance".

17 Cette réponse par l'histoire, il ne la souhaite pas car elle met en défaut la transcendance de l'art, et l'universalité du Musée. En revanche, il revendique clairement l'historicité de cette transcendance, lorsqu'il soutient que "aucune civilisation, avant la nôtre, n'a connu le monde de l'art créé par les artistes pour qui l'idée d'art n'existait pas". Il va plus loin dans La Création artistique: "Et s'il existe des oeuvres exceptionnellement durables, il n'existe pas plus de style éternel que de style neutre. Celui qui proclame le primat éternel d'un style se veut donc hors de l'histoire. "L'universalité de l'art, telle qu'il la conçoit, est une vision contemporaine et rétrospective. Il développe longuement d'ailleurs le 
caractère substitutif de cette vision au christianisme et sans cesse, on ressent que pour lui l'art a quelque chose à voir avec le salut. Tout en affirmant la transcendance comme datée, il s'éloigne du "jansénisme"qui croit que certaines oeuvres sont prédestinées, par leur forme, à devenir des oeuvres d'art.

L'art n'est pas nécessairement déposé dans l'objet, de même que le sens, dirait Michel Foucault, n'est pas déposé dans les choses. Tout objet, profane ou sacré, peut être un jour inscrit dans le musée imaginaire, dès qu'il se met à parler à l'imagination d'un être humain. La contrepartie de ce théorème est qu'il existe des oeuvres d'art sans artiste, du moins sans artiste conscient de faire œuvre d'art, et que, inversement, un artiste qui produit délibérément (ce qui veut dire dans notre idéologie" librement"- qu'on interprète ce" librement "comme ayant rapport au libéralisme économique ou à la liberté d'expression) une œuvre pour lui nécessairement pleine de sens dès sa création, pourra un jour, ou ailleurs, tomber dans l'insignifiance. Ils peuvent redevenir objet comme le carrosse redevint citrouille. Telles ces sculptures contemporaines dont un jardinier suisse nettoya le jardin public qu'elles encombraient, ou ces architectures naguère pensées, qu'on détruit à coup d'explosifs.

Même si le Musée imaginaire accorde une place majoritaire aux objets sacrés, Malraux n'en exclut pas par principe les autres : "Notre découverte de la signification des styles sacrés ne fait nullement du sacré la référence de tout art". L'éclectisme triomphal que Malraux affiche dans ses idées sur l'art, où se côtoient objets profanes et sacrés de toutes provenances, il l'attribue à la découverte des arts anciens ou exotiques permis par les progrès de la photographie, de la reproduction ("les arts plastiques ont inventé leur imprimerie"), conjoint au développement des voyages ("la confrontation d'un tableau au Louvre et d'un tableau à Madrid ou à Rome était celle d'un tableau et d'un souvenir"). Curieusement il ne saisit pas le lien avec l'ouverture démocratique, que Zola avait fait au Salon de 1887, lorsqu'il déclarait que la peinture était devenue une vaste confiserie avec des bonbons pour tous les goûts.

Après une telle ouverture du Musée imaginaire, on pourrait s'attendre à une analyse de l'épaisseur sociale de l'art. Elle ne vient pas, pas plus qu'elle ne vient dans son action militante. D'ailleurs, le trivial l'agace: "Vermeer n'a pas entrepris d'éterniser une laitière ! "Il se conduit avec l'art comme en politique, revendiquant sa mission sociale sans jamais en parler. Il ne s'engage que dans des combats d'idées qui survolent les questions concrètes, toujours sacrifiées. Sa conception de l'art, même s'il l'affirme enracinée dans l'homme et non nécessairement liée au sacré, demeure" le contraire d'une histoire de l'art".

21 Comme dans son action, il ne trouve sa dimension politique qu'au niveau international. L'art, au sens où il l'entend d'une construction historique de l'imaginaire, est clairement pour lui l'effet de la mondialisation: "Ce n'est pas notre perspicacité, qui nous révèle dans les plus grands artistes des civilisations disparues, le pouvoir tantôt délibéré, tantôt subordonné, tantôt ignoré d'eux-mêmes (et effacé pendant des siècles) par lequel leurs oeuvres nous atteignent: c'est l'entrée en scène de l'art mondial". Ces phrases sur la mondialisation de l'esthétique, aujourd'hui que la mondialisation de l'économie s'installe, prennent une autre consonance, que Malraux n'avait qu'entrevue. 
Malraux récuse l'esthétique unique, celle des civilisations religieuses, monarchiques ou totalitaires. Pourtant, l'ensemble de ces esthétiques venues du fond des siècles et des cinq continents, réunies dans le Musée imaginaire, communient pour lui dans un phénomène unique, inventé par le monde moderne, qu'il appelle l'art. Plutôt qu'à un polythéisme, il faudrait comparer la vision de l'art chez Malraux à un animisme. L'art, décidément, est un flux, un souffle qui parcourt l'Histoire et fait vivre ou revivre les objets pour les arracher au chaos.

Le Musée imaginaire et l'Inventaire général partagent un autre privilège: celui de contempler les oeuvres in situ, avantage essentiel pour la compréhension de l'art, sur le musée. Malraux, tout amateur de musées qu'il fût, en suspecte toujours l'incomplétude et la contingence qui préside à l'idée de collection. Il parle du musée comme d' "un palais où l'art semble avoir pénétré comme une dépendance de l'ameublement". Plus encore, il regrette la dénaturation qu'impliquent le passage au musée et le déracinement de l'œuvre : "Le musée transforme l'œuvre en objet: que l'on compare les salles gothiques du Louvre et même le musée des cloîtres de New York avec une cathédrale! Alors que le Musée imaginaire ajoute à chaque vrai musée (outre ce que possèdent tous les autres), la cathédrale, le tombeau, la caverne qu'aucun d'entre eux ne pourrait posséder".

En tenant ainsi ouvert sur le monde et sur l'histoire le grand registre des oeuvres d'art, André Malraux n'engageait-il pas le ministre de la culture qu'il devint à s'en faire le greffier ? Certainement pas pour que l'Etat soit le Dieu de ce jugement dernier, comme les musées, lorsqu'ils se prononcent sur leurs acquisitions imprescriptibles, mais en dressant l'inventaire de tous les musées possibles, à une époque donnée.

"L'art ne s'apprend pas, il se rencontre". "Nul ne sait où l'art prend ses sources". De telles phrases semblent préparer un Inventaire général. La découverte de l'art est moins affaire de découvertes d'objets, cachés ou ignorés, qui appartiennent déjà au monde de l'art, que de découvertes de terres inconnues, d'un élargissement du spectre de l'intelligence et de la sensibilité humaines, à des objets déjà connus mais dont la qualité esthétique est voilée, voire refusée. Malraux emploie l'image de la "cataracte" dont nos yeux, en matière d'art, sont perpétuellement affectés pour expliquer que l'Occident n'ait ajouté que si récemment les objets d'autres civilisations à son propre imaginaire.

Si telle est l'idée de Malraux, l'Inventaire général répond à ses attentes. L'Inventaire général n'a découvert que peu de chefs d'oeuvres oubliés. Il n'a retrouvé ni la trace du Michel-Ange du château de Bury, ni celle des Puget du château de Lalonde. L'Inventaire général ne permettra pas d'ajouter une aile nouvelle au Louvre. Mais il permettra d'ouvrir de nouveaux Louvres: celui de l'art industriel, celui de l'art ordinaire (qui répond à un ordre oublié), celui des objets scientifiques considérés sous leur aspect esthétique, celui des arts aujourd'hui réprouvés (jadis officiels), des modèles et de leurs copies, des arts déprisés ou, pour parler comme Hérodote, "des oracles qui ont cessé", ou encore, pour paraphraser Malraux, de ces oeuvres qui n'intéressent pas plus aujourd'hui les amateurs que Manet n'aurait intéressé Giotto. 
L'ection au "monde de l'art" d'objets qui n'y sont pas reconnus suscite naturellement des débats, souvent polémiques. C'est le fonctionnement même d'une pratique démocratique de l'esthétique. Certains s'irritent et voudraient plutôt que la valeur de l'art fût inflexible, fondée sur une qualité des oeuvres qui serait incontestable, mais dont ils resteraient les juges. Ils redoutent la confusion des hiérarchies, qui mettrait à égalité maîtres et épigones, chefs-d'oeuvre et oeuvres secondaires. L'industrialisation de l'art et sa mondialisation ont beaucoup fait pour entretenir cette inquiétude. L'Inventaire général par son esprit systématique peut y contribuer, comme l'étude savante des Ecritures saintes inquiétait l'Eglise. Malraux de ce point de vue, n'est pas Renan et observe un respect absolu de la vulgate.

La hiérarchie entre deux objets, d'ailleurs, est naturelle et existera toujours : entre deux signes l'un paraîtra toujours plus significatif que l'autre. Le culte de la différence n'est pas propre au monde de l'art : celui du sport le mesure en fractions de secondes et fonde, comme en art, un vedettariat toujours fragile. Dans un espace de valeurs homogènes, il n'est pas interdit de dire que Rembrandt est un plus grand graveur que Lievens et Lievens meilleur que Bol, si l'on s'entend sur les critères de jugement retenus. Ce qui est en fait redouté, c'est la lumière jetée sur l'origine de ces jugements de valeurs, qu'on veut garder mystérieuse en les croyant fondés sur un ordre incontestable et éternel, naturel ou surnaturel. La lumière apporte le doute et le doute est toujours un danger pour celui qui vit de certitudes.

Il n'y a pas de contradiction entre la vénération de Malraux pour les célébrités de l'art et sa volonté de ne pas considérer le palmarès comme intangible. André Malraux peut bien laisser ouvertes les portes de l'art, sans renoncer à vanter les seuls maîtres et les seuls chefs-d'œuvre, puisqu'il les consacre dans un musée dont il reconnaît la contingence historique et le caractère singulier. L'Inventaire général ne menace aucun ordre établi, mais il peut aider à comprendre l'ordre établi. Qui fait l'art, les artistes, les triomphes, les décadences et les redécouvertes? Il ne suffit pas de constater les variations du goût, chose assez évidente pour devoir être admise, il faut en expliquer les raisons, si la raison en est humaine. L'établissement des jugements de valeur, qui constitue la seule histoire de l'art, passe par l'analyse complète de leurs échelles.

Comme Roger Caillois, la pléthore nous effraie. Nous supportons mal l'idée que nous sommes entourés d'objets d'art qui sommeillent, que seule notre sensibilité et notre intelligence peuvent éveiller. On craint que l'œuvre unique, ou exceptionnelle, ne soit dévaluée ou corrompue par le nombre. Qui ne voit la métaphore du monde social qui se joue dans ces peurs symboliques du monde de l'art? La mise en perspective des oeuvres majeures par leur descendance ou leur parentèle n'a pas que des effets multiplicateurs. Elle change l'optique et l'appréciation de l'œuvre même qui n'apparaît plus isolée mais constituée de l'ensemble des membres de sa famille. Cet effet commence à être pris en compte par les historiens qui admettent qu'une architecture, une photographie, un livre, un tableau même, toute œuvre reproductible, ne peut être comprise ou appréciée tant que l'on n'en a pas embrassé les variantes.

31 Les oeuvres de série qui peuplent l'Inventaire général ne mettent pas en péril la conception de l'œuvre unique mais au contraire, on le constate chaque jour, en renforcent le prestige. André Malraux n'a pas commis l'erreur de jugement de Walter Benjamin qui a cru que la reproductibilité des oeuvres d'art affaiblirait leur "aura". Tout prouve aujourd'hui le contraire, même si l'on n'est pas certain qu'il en sera encore 
longtemps ainsi. Le système du vedettariat et le culte de l'original - les prix en témoignent - n'a jamais si bien fonctionné dans le monde de l'art. La reproductibilité technique des oeuvres, thème majeur du "Musée imaginaire", a renforcé la notion de modèle sans laquelle aucune série n'est possible. L'image de l'art prolonge son caractère sacré, comme dans la religion l'image nous rapproche des dieux en même temps qu'elle nous tient en respect.

Rien n'interdit d'admettre dans le Musée imaginaire de Malraux des objets ordinaires, des objets profanes, des objets industriels, des objets fonctionnels qui auraient troqué leur usage premier pour un usage symbolique. Même si l'on ne rencontre, à l'appui de ses propos, qu'une grande majorité d'oeuvres religieuses, il affirme ne rejeter en rien l'objet utilitaire au moment où il perd son utilité. Le fonctionnalisme d'ailleurs n'est pas un critère d'exclusion du monde de l'art, et beaucoup d'artistes majeurs du XIXe et du XXe siècles s'en sont fait une règle. Si l'on suit Malraux, l'Inventaire général fonctionnerait moins comme une "réserve" du musée imaginaire que comme une "avant-garde", au sens que ce terme a pris dans le monde de l'art. En sélectionnant parmi les objets du passé et ceux qui n'appartiennent pas encore au monde de l'art, tous ceux dont il juge qu'ils ont un caractère exemplaire, il contribue à enrichir le musée autant que chacun veut bien y reconnaître son bien, sa famille, et poursuivre le dialogue.

Sans être contradictoire avec l'œuvre de Malraux, l'idée d'un Inventaire général ne lui est pas moins étrange, comme si la scène se jouait sur un autre registre, ou si l'on en voyait les coulisses. Sans doute l'érudition éclectique et la curiosité inépuisable d'André Chastel furent-elles pour beaucoup - disons même l'essentiel - dans la conception de l'Inventaire général. Les circonstances de l'après-guerre y furent pour autant, que Chastel évoque ainsi : "Une espèce de grand silence suit le vacarme des cataclysmes : l'art n'avait plus de sens, à moins d'appartenir au destin, de devenir destin, comme Malraux allait le formuler". Sans les appels d'André Chastel, et la caution morale et intellectuelle de Julien Cain, Malraux, seul, n'en aurait peut-être pas eu l'idée, ou simplement l'audace. Mais, tout bien considéré, l'entreprise savante et contrôlée de l'Inventaire n'est pas opposée aux élans de son créateur, pour qui l'art n'est jamais donné d'avance, et, comme l'amour, n'est pas affaire de beauté, mais de passion.

Une seule condition aurait pu faire condamner l'entreprise par Malraux : ses limites aux frontières de la France. Certes, cette condition administrative et politique épousait à merveille les ambitions gaulliennes et s'inscrivait fatalement dans les programmes de l'après-guerre auxquels Malraux adhéra. La pertinence scientifique d'une telle limite frontalière doit cependant être mise en cause, et le sera nécessairement au regard de l'histoire européenne. Mais on ne peut s'empêcher de penser, en relisant Malraux, que, s'il n'avait tenu qu'à lui, il aurait commandé l'Inventaire général de l'humanité. 
INDEX

Mots-clés : en ligne, France, histoire de l'art, inventaire général, journal, patrimoine, périodique, revue électronique, revue numérique

Keywords : ejournal, electronic journal, heritage, history of art, on line

\section{AUTEUR}

\section{MICHEL MELOT}

Conservateur général des bibliothèques. Chargé de la sous-direction des études, de la documentation et de l'Inventaire, Hôtel de Vigny 10, rue du Parc Royal 75003 Paris. michel.melot@culture.gouv.fr 